\title{
HISTOCHEMICAL DISTRIBUTION OF PHOSPHORYLASE IN THE BRAIN OF THE GUINEA-PIG
}

\author{
BY \\ REINHARD L. FRIEDE \\ From the Aero Medical Laboratories, Wright Air Development Center, Dayton, Ohio, U.S.A.
}

The histochemical distribution of phosphorylase activity in brain tissue of rodents was described by Shimizu and Okada (1957) using fresh, frozen tissue sections. The present article describes the distribution of phosphorylase activity in the brain of the guinea-pig as revealed by using tissue slices trimmed by hand instead of sections for incubation (Friede, 1956). This technique provides an improved localization of phosphorylase activity. The preparation of tissue for incubation seems to be a critical phase of the reaction since changes in the enzyme activity result from tissue damage (Friede, 1959a).

The distribution of phosphorylase in brain tissue as defined by this technique will be compared briefly with an available large sampling of the distribution of succinic dehydrogenase in brain tissue. An inverse relationship between the two enzymes would be conceivable, considering the former one as a link of the anaerobic glycolytic breakdown and the latter as an indication of the presence of an oxidative Krebs cycle.

\section{Material and Methods}

Phosphorylase activity was demonstrated in 0.5 to $1 \mathrm{~mm}$. thick hand-cut tissue specimens prepared from the brain as quickly as possible after killing the animal. Extreme care was used to prevent any mechanical damage to the tissue. The specimens were incubated for three hours at $36^{\circ}$ to $38^{\circ} \mathrm{C}$. in the following medium:

$\begin{array}{lll}\text { Glucose-l-phosphate } & \ldots & 6 \% \\ \text { Glycogen } & . . & 0.1 \% \\ \text { Adenosinetriphosphate } & \ldots & 1 \% \% \\ \text { Mono iodacetate } . . & . & 0.25 \%\end{array}$

During the incubation, glycogen was synthesized from Cori ester; the added glycogen served as a starter of the reaction, but its concentration was too low to appear histochemically in the sections. No pre-existing glycogen could be found in brain tissue under these conditions. Mono iodacetate essentially improved the reaction by blocking the glycolytic breakdown of the Cori ester. Adenosinetriphosphate (or adenylic acid) activates phosphorylase (Takeuchi and Kuriaki, 1955). The solution adjusted itself to a $p \mathrm{H}$ of $7 \cdot 0$, and the addition of buffer to obtain the optimal $p \mathrm{H}$ or of inorganic ions did not essentially influence the results. Glucose and phlorhizin inhibited the reaction. No glycogen was synthesized by substituting the Cori ester with glucose-6-phosphate, glucose, fructose-6-phosphate, or fructose-1,6-diphosphate.

After the reaction was completed the specimens were fixed in Carnoy's fluid and embedded in paraffin. Glycogen was demonstrated by the periodic-acid-Schiff reaction; it was identified by diastase controls.

Some 130 incubations were used for the present investigation, each containing at least three specimens from various regions of the brain. In addition, one guinea-pig brain was studied completely by serial sections, $12 \mu$ thick. Alternate sections were stained with periodicacid-Schiff, Einarson's chromalum gallocyanin, and a combination of both stains.

\section{Results}

White Matter.-White matter generally exceeded grey matter in phosphorylase activity. The axons in the white matter were demonstrated and found to give an intense phosphorylase reaction. A granular reaction between the axons was also found. Most of these granules seemed to result from varying intensity of the reaction in the axons or from transversally cut axons. Phosphorylase activity was rarely localized in glial cells although it should be easy to recognize it at this site. The phosphorylase activity of white matter, therefore, seemed predominantly localized in the axons.

The phosphorylase activity of large fibre systems was more marked than that of the surrounding tissue; for example, the pyramidal tracts, the descending trigeminal root, the lateral tracts of the medulla oblongata, and the entire cerebral and cerebellar white matter showed strong activity. The medial longitudinal fascicle exhibited phosphorylase activity in its large axons. By contrast, the tractus solitarius always stood out by the negative reaction of its surrounding nucleus. The roots of the cranial nerves and the optic fascicle exhibited strong phosphorylase activity in their large axons. A very strong reaction was found in the retroflex tract of Meynert. 
Gray Matter.-The inconsistency of the phosphorylase reaction in neurones was studied in a previous article (Friede, 1959a). Strong phosphorylase activity was found in the chromophilic, shrunken neurons; however, no activity was found in the swollen, chromatin-depleted neurons. Since these neuronal changes evidently were post-mortem changes, the significance of a positive reaction is open to discussion.

In the present material, neurons with strong phosphorylase reaction were found irregularly and scattered in almost any nucleus; they were frequently found in damaged tissue portions near the surface of the specimen.

The neuropil of the brain's nuclei, as a rule, lacked phosphorylase activity, especially if mechanical damage was minimized during preparation. This negative reaction of the neuropil did not result from inadequate diffusion of the incubation medium into the specimen, since transfixing axons were sharply outlined by a contrasting strong reaction. A delicate network, demonstrated by phosphorylase activity, was occasionally found in the neuropil. It could not be established whether this reticulum was formed by finer axon or dendrite branchings or by glial processes, but the latter seemed less probable. Phosphorylase activity in glial cells may occasionally be found in gray matter, but did not constitute a typical feature. Gray matter exposed to mechanical damage, such as at the cutting surface, showed very strong phosphorylase activity by abundant glycogen deposition. Other nuclei immediately beneath the specimen's surface showed no reaction if not injured by the cutting. The diffusion conditions in such nuclei, however, were similar to those at the cutting surface, as proven by the heavy reaction in transfixing axons.

The above description fits most of the nuclei of the medulla oblongata, pons, mesencephalon, and diencephalon. Weak phosphorylase activity was particularly found in the dorsal vagal nucleus, the nucleus of the tractus solitarius, the locus caeruleus, and the pars compacta of the substantia nigra. These nuclei are mentioned because of their comparative histochemical interest as discussed later.

Some superficial nuclei, such as the nucleus prepositus hypoglossi, the medial vestibular nucleus, the cochlear nucleus, and the mammillary body, frequently, but not consistently, exhibited strong phosphorylase activity in their neuropil. Both exposure to mechanical damage during preparation and optimal diffusion conditions may contribute to this reaction.

Phosphorylase activity was consistently found in the molecular layer of the cerebellar cortex, the superficial layers of the acoustic tubercle, and the anterior quadrigeminal body, the upper layers of the cerebral cortex, and the caudate nucleus. The cerebellar cortex exhibited phosphorylase activity in the neuropil and in the large Purkinje dendrites of its molecular layer, but the reaction in the dendrites disappeared where they entered the cell body. Thus most of the cell bodies reacted negatively, but some of them showed strong phosphorylase activity. Weak activity was found in the cerebellar granular layer. The interpeduncular ganglion and the habenula showed in their neuropil the strongest reaction of all the brain's nuclei.

It is not clear whether this consistent phosphorylase reaction in some nuclei reflects activity in vivo or if these regions are extremely sensitive to damage and should show no phosphorylase reaction as other nuclei do if completely undamaged.

The periventricular gray matter of the aqueduct and the tuber cinereum frequently exhibited phosphorylase activity in astrocytes besides a diffuse granular reaction of the neuropil. No other region of the brain regularly showed phosphorylase activity in glial cells, though it was found at times.

Ventricular Walls and Leptomeninges.-A very strong phosphorylase activity was found in the subependymal glial tissue. Contrasting with this strong subependymal reaction, the ependymal cells lacked phosphorylase activity although in direct contact with the incubation fluid. Some glycogen droplets, however, were frequently found in the ventricular lumen, attached to the surface of the ependymal cells. Since synthesis of glycogen in this location did not seem likely, this finding may suggest a supravital transportation or secretion. The epithelium of the subcommissural organ, which is a specific ependymal formation in the aqueduct, completely lacked phosphorylase activity, but the glial tissue of the raphe immediately caudal to it exhibited an excessive reaction. The area postrema exhibited strong or mild activity. Somewhat variable strong to mild activity was also found in the leptomeninges.

Comparison of Distribution of Phosphorylase and Succinic Dehydrogenase.-Dempsey and Wislocki (1944) and Bradfield (1951) suggested that glycogen deposition in tissue was correlated with a high anaerobic metabolism. An inverse relationship between the distribution of glycogen deposition and the distribution of succinic dehydrogenase and cytochrome oxidase in the periventricular structures of the brain was observed by Shimizu, Morikawa, and Ishi (1957). The above findings, therefore, were compared with an available large sampling of the distribution of succinic dehydrogenase in brain tissue of the guinea-pig (Friede, 1959b). Succinic dehydrogenase was demonstrated in this material in 


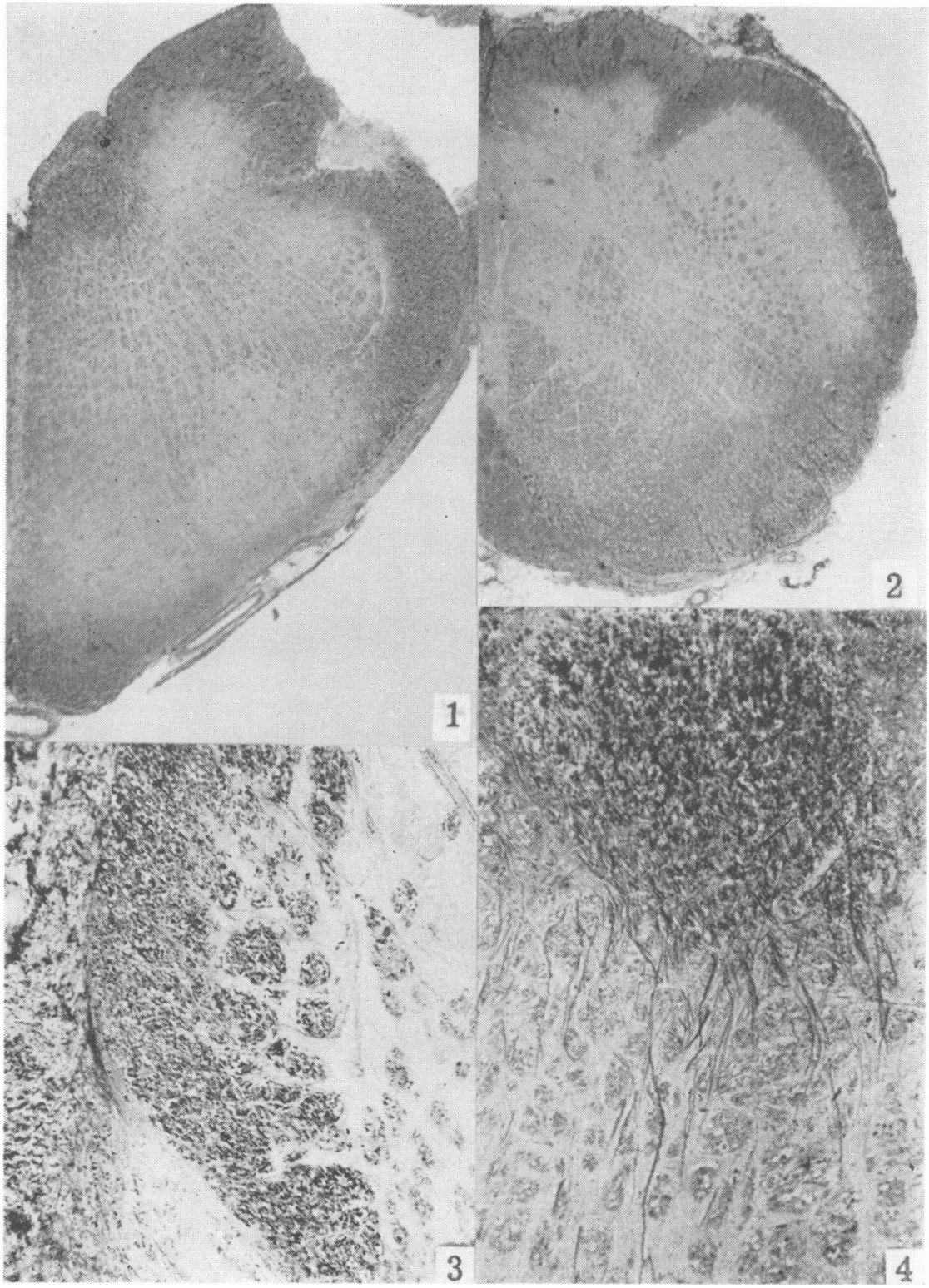

FIG. 1.-Distribution of phosphorylase activity in the medulla oblongata. The lateral vestibular nucleus, the nucleus of the descending trigeminal tract, and the facial nucleus stand out as light areas. Periodic-acid-Schiff $\times 16$.

Fig. 3.-Descending trigeminal root (middle). Fibre bundles and transfixing axons clearly stand out on the negative reaction of the nucleus of the descending trigeminal root (right). The peripheral fibres (left) show a weaker reaction than the descending trigeminal tract. Periodic-acidSchiff $\times 80$.
FIG. 2.-Lower medula oblongata, showing a negative reaction in the nuclei of the dorsal fasciculi and the spinal trigeminal tract and a very faint reaction in the hypoglossal nucleus. The space of the facial nucleus (Fig. 1) is occupied by heavily reacting fibre tracts. Periodic-acidSchiff $\times 16$.

Fig. 4.-Genu of the facial nerve showing both transversally and longitudinally cut fibres exhibiting strong phosphorylase activity. Periodicacid-Schiff $\times 80$. 


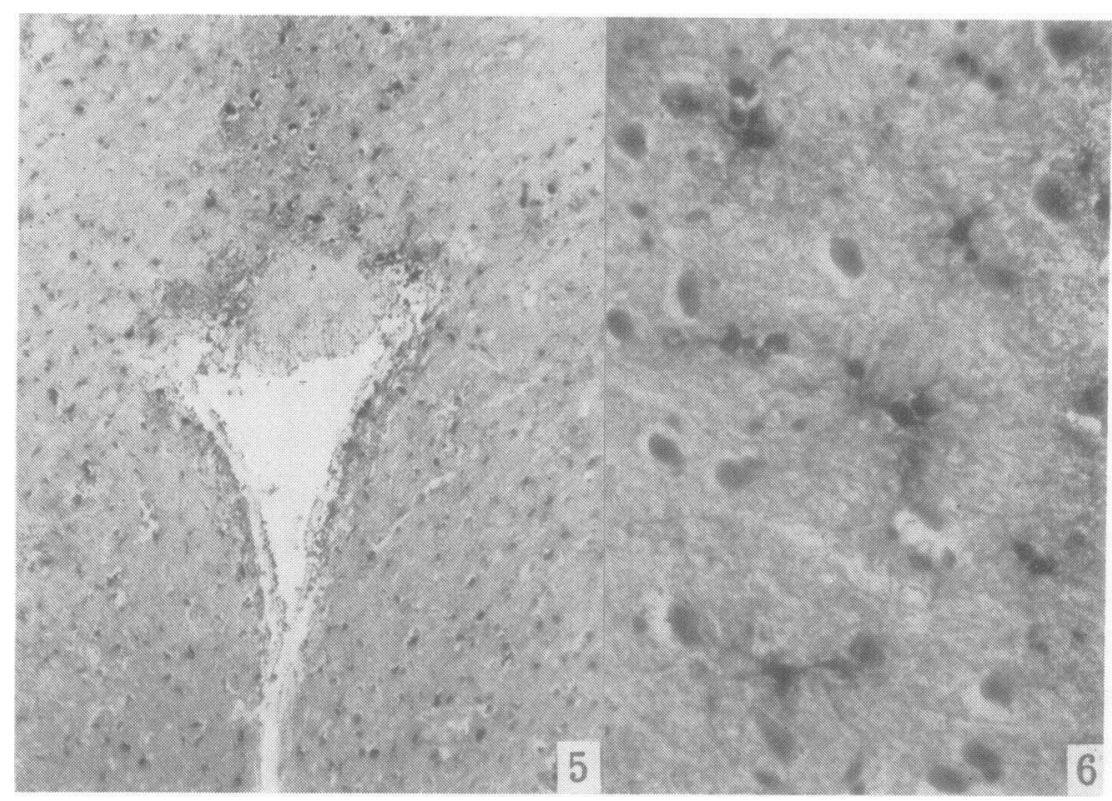

Fig. 5.-Oral portion of the aqueduct. The subcommissural organ and the ependyma show no reaction; a strong reaction is found in the subependymal glial tissue, and mild, diffuse reaction in the central gray matter. Periodic-acid-Schiff $\times 80$.

Fig. 6.-Lateral part of the tuber cinereum showing phosphorylase activity in glial cells. A faint ccunterstaining by chromalum gallocyanin shows neurons without reaction. Periodic-acid-Schiff $\times 320$.

frozen sections of fresh tissue, $60 ; i$ thick, prepared by the author's technique (Friede, 1958b). (This article may be consulted for further details.) Both "tetrazolpurpur", Bayer (4,4-bis 3,5-diphenyl-2tetrazoliumbiphenyl) chloride (Neumann and Koch, 1953) and "nitro BT" (Nachlas, Tsou, De Souza, Cheng, and Seligman, 1957) were employed.

Strong phosphorylase activity and weak or no succinic dehydrogenase activity coincided in the white matter, the subependymal glial tissue, and the glial tissue of the raphe.

Weak phosphorylase activity and strong succinic dehydrogenase activity coincided in the ependymal cells and in neurons and neuropil of many of the brain's nuclei. Many other nuclei, however, showed mild or strong phosphorylase activity instead of their strong succinic dehydrogenase activity. In such nuclei an inverse relationship of the enzymes may nevertheless be present, since a loss of succinic dehydrogenase has been observed during the incubation for phosphorylase in portions of the specimens which were exposed to mechanical damage and exhibited strong phosphorylase reaction (Friede, 1959a).

An inverse relationship was not evident in glial tissue. Glial cells seem to contain none or weak succinic dehydrogenase activity. A consistent phos- phorylase reaction, however, was found only in the glia of the periventricular gray matter and in the subependymal glia.

No inverse relationship was found in the subcommissural organ which lacked both phosphorylase and succinic dehydrogenase activity. Moreover, the nucleus of the tractus solitarius, the dorsal vagal nucleus, the locus caeruleus, and the pars compacta of the substantia nigra exhibit rather weak phosphorylase activity and none or weak succinic dehydrogenase activity (Shimizu and Morikawa, 1957).

An inverse relationship of the enzymes is lacking also in the developing rat's brain, which biochemically (Potter, Schneider, and Liebl, 1945) and histochemically (Friede, 1959b) exhibits weak succinic dehydrogenase activity but also shows biochemically (Shapiro and Wertheimer, 1943) and histochemically (Shimizu and Okada, 1957; Friede, 1958a) weak phosphorylase activity.

\section{Discussion}

Phosphorylase activity in white matter has been demonstrated histochemically in both fresh frozen tissue sections (Shimizu and Okada, 1957) and in hand-cut tissue specimens. In the latter, the localization of the enzyme activity was more detailed 
than the diffuse reaction in sections; the gross distribution, though, was similar. The evaluation of the findings in gray matter, however, is most difficult. The reaction in tissue sections was reported as being highly labile (Shimizu and Okada, 1957). In tissue specimens, a strong reaction in gray matter seemed to depend on tissue damage during the preparation. Weak or no phosphorylase activity was found in most nuclei especially if any tissue damage was avoided, including cutting of sections. The weak or negative reaction of gray matter was not simulated by inadequate diffusion conditions within the tissue specimen since transfixing axons in the gray matter showed typical strong phosphorylase reaction. It does not seem likely that the reaction is weakened in gray matter by a competitive consumption of the glucose phosphate, since the glycolysis was blocked by iodoacetate, and since the reaction of the transfixing axons would be weakened as well.

A strong phosphorylase activity in neurons was found to be accompanied with artificial cell shrinkage (Friede, 1959a). The physiological phosphorylase activity of gray matter, therefore, seems rather low, but relatively slight tissue damage seems to activate the enzyme which may be present in an inactive stage. In the first 30 seconds of anoxia, as an example, glycolysis was reported increased to 10 times the aerobic glucose utilization (Opitz and Schneider, 1950).

It is not clear whether some regions of gray matter which consistently show phosphorylase activity (as the upper portions of the cerebral cortex or the molecular layer of the cerebellar cortex) are extremely sensitive to a preparative damage or physiologically if they exhibit enzyme activity. The very strong phosphorylase activity found in the habenula and the interpeduncular nucleus may bear a relationship to the outstanding activity of alkaline phosphatase in these nuclei (Shimizu, 1950).

The activation of phosphorylase by tissue damage may explain the much higher activity rates found in gray matter by biochemical techniques involving tissue destruction: in the cerebellum, phosphorylase activity of 2.79 units in the molecular layer, 2.76 units in the granular layer, and 1.62 units in the subjacent white matter was found (Robins, Smith, and Jen, 1957). These results are in contrast to the weak phosphorylase activity found in the granular layer by histochemical techniques. Sensitivity to tissue damage probably also explains why it is possible to obtain a cytochemical demonstration of an enzyme which has a very poor structural binding if investigated by biochemical techniques.

An inverse relationship of phosphorylase activity and succinic dehydrogenase activity is generally found, though exceptions are not rare. This inverse relationship may be considered indicative for different aerobic or anaerobic metabolic rates.

\section{Summary}

The histochemical distribution of phosphorylase activity in the brain of the guinea-pig is described with a technique providing an improved localization of the enzyme activity. Strong phosphorylase activity was found in axons in both white and gray matter. Rather weak activity was noted in the neuropil and neurons of gray matter. Even extremely slight tissue damage was found to alter the reaction. The histochemical distribution of phosphorylase activity and succinic dehydrogenase activity generally was inverse but a few exceptions were noted in particular regions.

\section{REFERENCES}

Bradfield, J. R. G. (1951). Nature (Lond.), 167, 40. Dempsey, E. W., and Wislocki, G. B. (1944). Endocrinology, 35,

Friede, R. (1956). Arch. Psychiat. Nervenkr., 195, 325.

- (1958a). Hoppe-Seyl. Z. physiol. Chem.. 310, 4.

(1958b). J. Histochem. Cytochem., 6, 347.

(1959a). Ibid., 7, 34.

(1959a). Ibid., 7, 34.

Nachlas, M. M., Tsou, K. C., De Souza, E., Cheng, C. S., and Seligman, A. M. (1957). J. Histochem. Cytochem., 5, 420.

Neumann, K., and Koch, G. (1953). Hoppe-Seyl. physiol. Chem.,

Opitz, E., and Schneider, M. (1950). Ergebn. Physiol., 46, 126.

Potter, V. R., Schneider, W. C., and Liebl, G. J. (1945). Cancer Res., 5, 21.

Robins, E., Smith, D. E., and Jen, M. K. (1957). In Ultrastructure and Cellular Chemistry of Neural Tissue, ed. H. Waelsch, p. 205. Hoeber-Harper, New York.

Shapiro, B., and Wertheimer, E. (1943). Biochem. J., 37, 397.

Shimizu, N. (1950). J. comp. Neurol., 93, 201.

- and Morikawa, N. (1957). J. Histochem. Cytochem., 5, 334 $\longrightarrow$, and Morikawa, N. (1957). J. Histochem. Cytochem

- , and Okada, M. (1957). J. Histochem. Cytochem., 5, 459. Takeuchi, T., and Kuriaki, H. (1955). Ibid., 3, 153. 